\title{
EL CAMINO QUINDÍO EN EL CENTRO OCCIDENTE DE COLOMBIA. LA RUTA, LA RETÓRICA DEL PAISAJE Y LOS PROYECTOS DE POBLAMIENTO
}

\author{
Álvaro ACEVEDO TARAZONA ${ }^{1}$ - Universidad Tecnológica de Pereira \\ Sebastián MARTÍNEZ BOTERO ${ }^{2}$
}

\begin{abstract}
RESUMEN: Muchas de las rutas que comunican las regiones del país fueron pensadas desde tiempos prehispánicos. Un gran número de los "caminos reales" que abrieron los españoles se hicieron sobre las rutas que ya tenían establecidas los aborígenes; sobre los caminos reales también se trazaron algunos tramos de las carreteras de la actualidad. Los caminos reales adquirieron esta connotación porque tanto su mantenimiento como administración se realizaba por delegación del rey. Fueron los más empleados durante la colonia porque servían para unir las provincias. Por comunicar el occidente con el oriente del país, el camino del Quindío es una de estas rutas que tiene antecedentes históricos desde los tiempos prehispánicos hasta la actualidad. Su historia está impregnada de nociones y representaciones sobre la naturaleza y la cultura que bien vale la pena indagar desde la perspectiva de la historia ambiental.
\end{abstract}

PALABRAS CLAVE: Camino del Quindío, nociones, representaciones, ambiente, naturaleza, cultura, viajeros, Colombia, España.

ABSTRACT: Many of the routes that communicate the regions of the country were thought from pre-Hispanic times. A great number of the "dirt roads" that opened the Spaniards did on the routes that already they had established the natives; on the dirt roads also some sections of the highways of the present(F. Zuluaga 1995:157). The dirt roads were denominated thus because as much their maintenance as administration was made by delegation of the king. They were used during the colony because they served to unite the provinces. To communicate the West with the east of the country, the way of the Quindío is one of these routes that have historical antecedents from the prehispanicos times to the present time. Its history is impregnated, in addition, by slight knowledge and representations on the nature and the culture that the pain is worth well to investigate from the perspe ctive of environmental history.

KEYWORDS: Way of the Quindío, slight knowledge, representations, ambient, nature, culture, travellers, Colombia.

\footnotetext{
${ }^{1}$ Profesor Universidad Tecnológica de Pereira, Doctor en Historia de la Universidad de Huelva, España.

2 Estudiante de Historia de la Universidad Industrial de Santander (Colombia). En la actualidad realiza su tesis de grado. Participó en el proyecto de investigación Cambios ambientales en perspectiva histórica en las cuencas medias de los ríos Otún y Consota de la Universidad Tecnológica de Pereira, 2002-2004.
} 
A Maria:

no te detengas en el camino.

"El camino era otra vez miserable.

Tuvimos tempestad con granizo. Pasamos muchas palmeras y en la lejanía detectamos altas montañas cubiertas de nieve"

Charles Stuart Cochrane, Diario de mi residencia en Colombia

"Esta empresa era pintada por algunos como fácil, pero los mapas manifestaban que era dificil; más no había otra salida" José Manuel Restrepo, Diario de un patriota

\section{LA RUTA DEL QUINDÍO}

El camino del Quindío fue uno los caminos reales que por siglos comunicó el occidente con el centro del país, y fundamentalmente el valle del Cauca con el río Magdalena y Santa Fé de Bogotá. Su ruta era también una prolongación del camino hacia Quito pasando por ciudades como Popayán, Cali, Buga, Cartago, entre otras. El historiador Francisco Zuluaga muestra las distancias que existían entre los diferentes tramos del camino durante el siglo XVI. Aquellas distancias también pueden dar una idea de la ruta que se seguía en ese tiempo, la cual tenía una longitud aproximada de 221 leguas (F. Zuluaga 1995:158)³:

Cuadro 1: Distancias y tiempos del recorrido Camino Real Santafé-Quito

\begin{tabular}{ccc}
\hline Tramo & Leguas & Días \\
\hline Santa Fé - Tocaima & 18 & 3 \\
Tocaima - Mariquita & 18 & 3 \\
Mariquita - Ibagué & 18 & 3 \\
Ibagué - Cartago & 22 & 4 \\
Cartago - Cali & 43 & 2 \\
Cali - Popayán & 22 & 5 \\
Popayán - Almaguer & 20 & 8 \\
Almaguer - Pasto & 20 & 7 \\
Pasto - Quito & 40 & $(15 ?)$ \\
\hline
\end{tabular}

${ }^{3}$ Francisco Zuluaga reconstruye el cuadro a partir de las siguientes fuentes: LÓPEZ DE VELASCO, Juan (1971). Geografia y descripción de las Indias, Madrid: Biblioteca de Autores Españoles-AtlasPassim; ANÓnImo (1983). «Relación de Popayán y del Nuevo Reino», en Cespedesia, suplemento. No. 4; pp. 23-103; NieTO, Diego Antonio (1983). «Visita de la Gobernación de Popayán, 1797», en Cespedesia, suplemento. No. 4; pp. 495-512; BARONA, Guido (1991). «Instrumento de consolidación del poder de Popayán, en sus relaciones con el valle de Neiva y el Reino de Granada», Ponencia presentada en el Congreso de Colombianistas, Ibagué. 
Si se comenzaba desde Popayán, avanzar sobre el camino del Quindío significaba dirigirse hacia Cali en donde se podía embarcar en un champán y navegar por el río Cauca, o bien seguir el curso de éste por un camino paralelo que terminaba en el "Paso de Gallo", donde se cruzaba el río La Vieja para ascender hasta Cartago la Antigua pasando por un pueblo de indios llamado Pindaná de los Cerrillos. Llegando a Cartago se ascendía la cordillera bordeando el curso del río Otún, se pasaba por un sitio llamado El Roble y luego se descendía para atravesar el río Quindío y llegar hasta otro sitio llamado Boquía. Se tomaba entonces el curso del río Quindío y se comenzaba a ascender nuevamente la cordillera hasta el río Toche por donde se encontraba el camino para descender a Ibagué. Una vez en Ibagué se tomaba el camino de Mariquita; allí se podía continuar hacia Santa Fé de Bogotá por la vía de Honda o tomar una embarcación en el Magdalena hacia la costa.

Cuadro 2: Relación de vecinos españoles, pueblos y habitantes indígenas en la ruta, $1560^{4}$

\begin{tabular}{cccc}
\hline Ciudad & $\mathrm{N}^{0}$. vecinos españoles & $\mathrm{N}^{0}$. pueblos indígenas & $\mathrm{N}^{\circ}$. indígen. tributarios \\
\hline Santa Fé & 55 & 57 & 36.552 \\
Tocaima & 31 & 59 & 3.201 \\
Mariquita & 23 & 36 & 2.038 \\
Ibagué & 27 & 36 & 2.701 \\
Cartago & 17 & 40 & 4.575 \\
Cali & 24 & 47 & 3.241 \\
Popayán & 19 & 31 & 8.659 \\
Almaguer & 23 & - & 3.260 \\
Pasto & 28 & 65 & 23.634 \\
Quito & - & - & 80.000 \\
\hline Total & 247 & 371 & 168.221 \\
\hline
\end{tabular}

Pero esta ruta no siempre fue permanente. Una de las variaciones del camino, precisamente, se dio con el traslado de Cartago en $1691^{5}$. Así, pues, ya no se

\footnotetext{
${ }^{4}$ Francisco Zuluaga reconstruye el cuadro a partir de las siguientes fuentes: «Relación Popayán y del Nuevo Reino, 1559-1560», Cespedesia, suplemento. No. 4 (1983); pp. 23-103.

${ }^{5}$ La ciudad de Cartago fue fundada por el Mariscal Jorge Robledo en 1540. Éste había sido enviado por el adelantado Sebastián de Belalcázar que venía conquistado tierras de sur a norte desde el Perú. Robledo terminó convirtiéndose en el gran descubridor del occidente del país, lo cual le mereció la muerte por orden del mismo Belalcázar. Cartago fue fundada en el sitio donde actualmente está ubicada la ciudad de Pereira y fue trasladada al sitio donde actualmente está ubicada el 21 de abril de 1691; por eso para referirse al lugar donde estuvo inicialmente, se dice Cartago la Antigua.
} 
tomaría el paso por Pindaná de los Cerrillos, "sino que de Cartago se salía a cruzar el río La Vieja por Piedra de Moler, y desde este sitio se construyó un nuevo camino que saldría al Roble, para luego descender a Boquía" (Zuluaga 2002:130131). Otra variación en la ruta se dio en 1856 cuando apareció la población de Salento (como consecuencia del traslado de Boquía) y el camino se desvió para cruzar por la nueva población.

La variante que se hizo para que el camino dejara de pasar por Pindaná de los Cerrillos, deja ver en las fuentes de archivo un pleito prolongado (Larrichio 2003). El pleito se basó en una discusión entre los cabildos de las ciudades de Cartago e Ibagué sobre cuál ruta debía renovarse: La Trocha que favorecía los intereses de Cartago, o la de los "Serrillos" que favorecía a los de Ibagué. La de los Serrillos era la ruta más conocida porque pasaba por Cartago la Antigua, pero La Trocha, que pasaba por el actual pueblo de Filandia (Quindío) y Alcalá -inicialmente llamado San Sebastián de la Balsa- (Valle del Cauca), al parecer era la ruta más corta.

A Larry Larrichio sus estudios de campo le demostraron que si bien la ruta de la Trocha era 10 a 15 kilómetros más corta, la de los Serrillos (Cerritos) tiene (1999 y 2001) todavía un relieve más parejo y plano que en su momento la haría de más fácil tránsito. De hecho en la actualidad es por esta ruta que pasa la carretera que comunica las ciudades de Pereira y Cartago, mientras que la de "La Trocha aún hoy no tiene camino pavimentado entre Filandia y Cartago, y las fincas de café tampoco tienen una arteria principal del oriente al occidente. Las veredas están conectadas por una red de caminos de herradura que durante la época lluviosa deja atascados a los vehículos" (2003). Sin duda para Larrichio el motivo de la disputa debió ser más político que cualquier otra cosa, pues si fuera por la topografía el problema se hubiera resuelto para que la ruta continuara por los Serrillos.

\section{EL DETERMINISMO DEL CAMINO: APUNTES PARA UNA POLÉ- MICA}

Se ha difundido una tendencia a creer que el camino del Quindío fue posible gracias a la dificultad por el paso del páramo de Guanacas, que se encontraba "infestado" de indios Pijaos, de los que entre otras cosas se decía que devoraban gente. Esta ruta cruzaba la cordillera al sur para comunicar a Popayán con Ibagué y Santafé, pasando por Timaná y La Plata y entrando por el Huila.

Investigaciones y leyendas han confirmado la tenaz resistencia de estos indios ${ }^{6}$,

\footnotetext{
${ }^{6}$ Hoy se sabe que tales indios no pertenecían estrictamente a los Pijaos sino a otras tribus que se refugiaban en las montañas huyendo de la avanzada hispana (Valencia Llano 1991:96).
} 
pero ello aún no demuestra que su hostil presencia en la cordillera Central haya sido determinante para interrumpir el paso por Guanacas y, en consecuencia, trasladar la ruta más al norte en tránsito por Cartago. Las guerras de pacificación fueron permanentes durante el periodo colonial y se presentaron en todas las regiones donde se concentraban los intereses hispanos. ¿Cuáles eran esos intereses? Básicamente dos: indios para encomiendas y minas para la explotación de oro. Si nos detenemos a mirar en dónde se hicieron las fundaciones de ciudades en el Nuevo Mundo, veremos que la mayoría de ellas se construyeron, y algunas aún permanecen, donde había asentamientos de indios, cerca de las minas o en los puertos.

Cartago no fue la excepción. Su fundador, el capitán Robledo, un andaluz (al parecer de Ubeda) en busca de fama y riquezas como la mayoría de hombres que pasaron a las Indias, levantó la ciudad de Cartago en medio de un territorio rodeado de grupos indígenas: Picaras, Paucuras, Carrapas, Pozos, Irras, Quimbayas, Quindos, Quninchias, Yuldanas, Purpomas, Burilas, Otaimas, Cacataimas, Anatomías, Gorrones, entre otros. De forma que los intereses oficiales cobraban vida en la medida que había mano de obra indígena que encomendar, y mejor si se encontraba cerca de las minas de oro o plata. Imaginar el territorio como una red de ciudades, tal como fue la intención del imperio español (Romero 1999), suponía además crear una dicotomía espacial: lo urbano contra lo rural; lo urbano como reflejo de la perfección y lo rural como tierra de nadie, salvaje, lo que hay que civilizar. Bajo esta lógica actuaron los primeros españoles y por ello fundaron ciudades.

Sabemos también que en esa carrera por afianzar el territorio, Robledo fundó en 1939 la ciudad de Santa Ana en el valle de Guarma, en donde estaba buscando sal entre los indios y quienes a las señas misteriosas del capitán, sólo respondían: "ANZER! ANZER!" (2002), por lo cual esta ciudad se identificaría mejor con el nombre de Anserma. "Efectuada la fundación se emprendieron expediciones con el fin de explorar la provincia. Ruy Venegas y Suero de Nava recorrieron el norte, mientras que el capitán Gómez Hernández atravesó la cordillera para explorar el Chocó" (Friede 2002:210). Cuando la fundación se puso en servicio de la expedición, se estaba cumpliendo lo estipulado por la tradición hispana de conquista.

Con esta misma lógica Robledo fundó Cartago el 9 de agosto de 1540 y Antioquia el 4 de diciembre de 1542. Al territorio de ésta última llegó en un intento frustrado por alcanzar la misteriosa provincia de "Arbi", por lo cual recorrió "Cenufana, exploró el valle de Aburrá, vadeó el río Cauca y alcanzó las minas de Buriticá, Ebéjico y Nori” (2002:251). La intención era muy clara, integrar estos 
nuevos territorios a los ya conquistados, antes de que alguien más los tomara, y apropiarse de las minas.

Para la fundación de Cartago se tuvo en cuenta "un lugar apropiado en el que hubiese buena agua, suficiente madera para construir casas, leña para fogones, buena caza y pesca, y crecido número de indios en su vecindad para el servicio de los futuros moradores" (2002:220). Cartago se fundó siguiendo la lógica hispana que presuponía que estar cerca de indios que encomendar iba a ser provechoso para los vecinos que se instalaran allí, y no podemos seguir pensando el pasado con la óptica que hoy nos guía, porque creer que la ubicación de Cartago era un enclave estratégico para quedar en el cruce de caminos, podría ser un peligroso anacronismo.

Si Cartago se benefició del tránsito de viajeros que por ella iban y venían de norte a sur, se debió principalmente a su breve esplendor aurífero en el siglo XVI y al auge minero que ocurrió en el siglo XVIII gracias al descubrimiento de las minas del Chocó, y del cual ciudades cercanas como Cartago comenzaron a servir de abastecedoras de las necesidades, sobre todo alimenticias, de las minas (Colmenares 1987:35-36). Lo cierto es que en la configuración de la provincia que alguna vez recorriera el capitán Robledo con su hueste, la ciudad de Cartago quedó ubicada en un lugar estratégico por estar entre el valle del Cauca y Antioquia. No obstante, esto no quiere decir que Cartago necesariamente dependiera de los caminos o que floreciera por ser ciudad de paso. De ser así, ¿cómo entonces se explicaría a finales del siglo XIX y comienzos del XX el relevo ocurrido de Pereira sobre Cartago, esta última una ciudad de paso que le llevaba más de trescientos años de ventaja a la otra? Podríamos asumir que la ubicación de una ciudad es estratégica, pero lo que determina su éxito o fracaso son las redes y connotaciones sociales que se tejen en torno a su territorio.

Cuando se comenzó a configurar la inmensa región que recorrió Robledo, surgió la necesidad de salir hacia el oriente, por un lado, para buscar el río Grande de la Magdalena que llevaba al mar, y por otro para comunicarse con la ciudad que fundara Jiménez de Quesada en 1538, Santafé de Bogotá, y sus ricas tierras de la altiplanicie. El primer español en intentar el paso por la cordillera Central fue el capitán Álvaro Mendoza, enviado por Robledo, quien fracasó "por la imposibilidad de cabalgar por la tupida selva, teniendo que hacer el trayecto a pie, desbrozando la montaña" (Estrada 1998:25). Pronto la cordillera cerraría completamente el paso del expedicionario y Mendoza tuvo que dar marcha atrás.

Esta "muralla" natural, sin embargo, sería traspasada, y el primer camino que se utilizó para comunicarse con el centro del país fue el de Herveo. El trayecto de este 
camino ascendía por el río Guarinó hasta los páramos de Herveo y el Ruiz para descender a Chinchiná y de allí hasta Cartago. Según Francisco Zuluaga este camino "brotó" con el fin de "permitir el traslado de oro de Mariquita para ser fundido y quintado en las Cajas Reales establecidas en Cartago desde 1542" (F. Zuluaga 1995:164). El camino adquirió importancia en la medida que se logró establecer relaciones económicas a través de él.

Mapa de caminos de herradura. Occidente y Noroccidente colombiano. Siglo XIX.

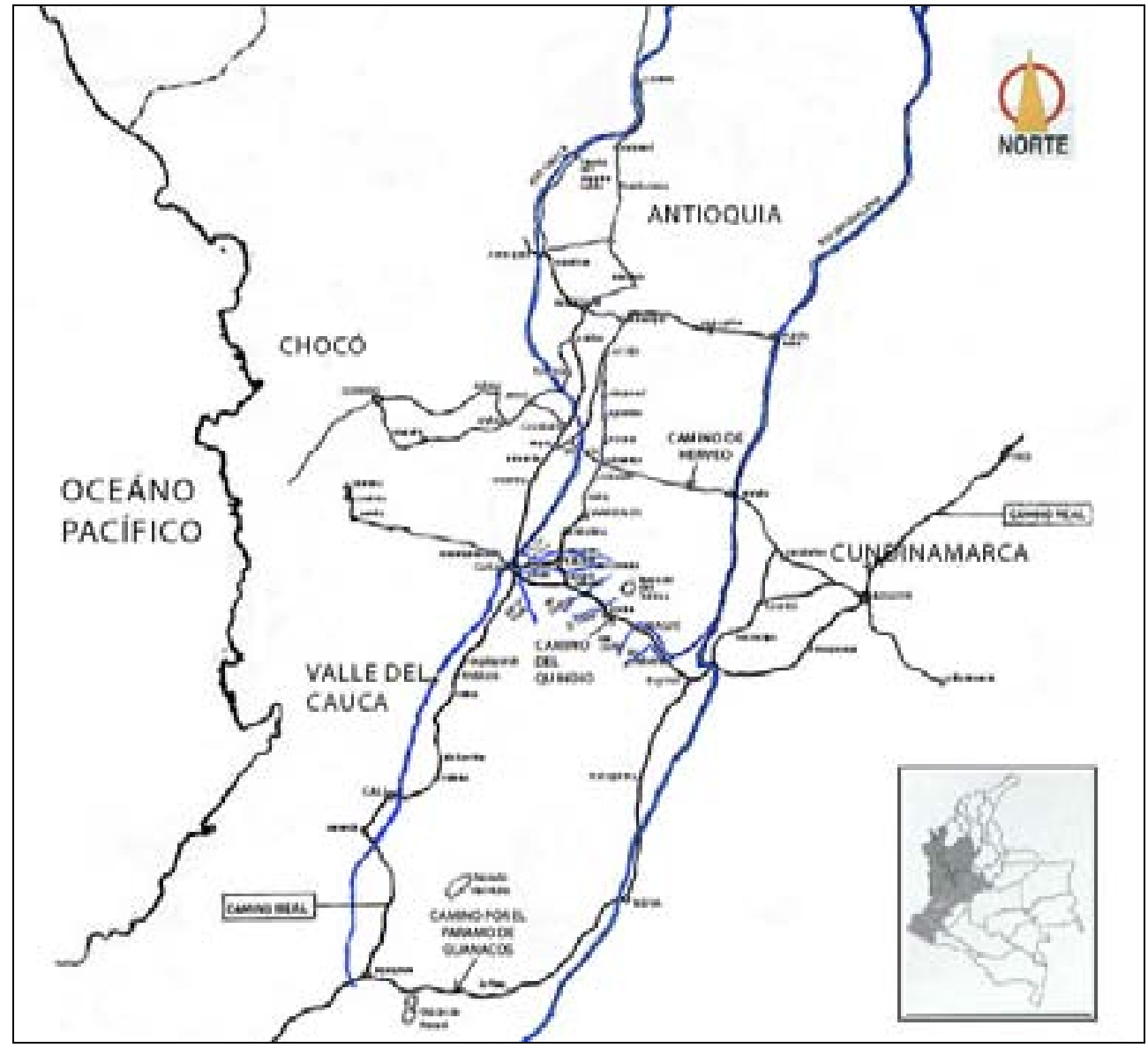

FUENTE: IGAC, Atlas de Colombia, 5 ed., Imprenta Nacional de Colombia, 2003. 
Esto mismo se entiende cuando el oidor Miguel Días de Armendáriz en 1547, refiriéndose al camino de Herveo, expresó: “... porque descubriéndose un camino de este reino a la ciudad de Cartago... se proveerá de este Reino allá carne y ropa de la tierra y sal, porque aquí es mucha la abundancia que hay de puercos y de lo dicho, y allá mucha falta, y a lo que se entiende mucha grosedad de minas; las cuales se dejan de seguir por la necesidad que dicen ser tanta..." (Friede 2002:287).

La importancia inicial de Cartago hacia 1547 tenía que ver con el hecho de constituirse como la primera frontera minera de la Gobernación de Popayán. Sebastián de Magaña, tesorero de la Real Hacienda, escribió por esa época al rey: "...donde más ricas minas ha habido y se cree que las habrá es en Cartago, Anserma y Arma y Antioquia...' (Carta de Sebastián de Magaña a su Majestad)" (F. Zuluaga 1995:164).

Recién fundada San Bonifacio de Ibagué por López de Galarza en 1550, uno de sus compañeros, Francisco Trejo, expresó que “...Luego como se pobló la dicha ciudad de Ibagué, tuvo noticia que pasada la cordillera del páramo estaba un pueblo de españoles que entendió era la ciudad de Cartago, fue por todo aquel despoblado y abrió el camino hasta llegar a ella y llevó caballos"'(1995:164-165) ${ }^{7}$. Mientras los vecinos de Cartago estaban utilizando el camino de Herveo para pasar al centro del país, la primera iniciativa de "abrir" el camino del Quindío provino de los vecinos de Ibagué, quienes esperaban obtener usufructo de ello. Por eso el Justicia Mayor de Ibagué, Melchor Valdés, "abre" el camino a sus expensas y la de los vecinos, aprovechando una depresión al sur de páramo del Quindío; camino que buscaba comunicarse con la población de Cartago, con el fin de que fueran los vecinos de Ibagué quienes gozaran de la "exclusividad en las 'arrias y recuas' para el transporte de las mercaderías" (1995:165).

Se dice que el camino que se abrió aprovechaba una ruta que ya empleaban los indios Quimbayas para cruzar la cordillera. Fabiola Estrada data el descubrimiento de esta ruta hacia el año de 1556, aunque tres años antes los hispanos ya estaban atravesándola, según testigos que refiriéndose a éste camino declararon que: "En todas las jornadas hay tambos de españoles, donde hacen noche los que por él caminan, y andan sin riesgo alguno" (Friede 2002:288). Juan Friede dice al respecto que desde que se fundó la ciudad de Ibagué "el mayor volumen del comercio con el Perú y Quito se efectuaba por él” (Friede 2002:288). Así es posible deducir que el camino del Quindío obedeció a una iniciativa de Ibagué más

\footnotetext{
${ }^{7}$ Francisco Zuluaga retoma la cita de COLMENARES, Germán (1973). Historia Económica y Social de Colombia, 1537-1719, Cali, Universidad del Valle.
} 
que de Cartago, pues el inicio de su servicio estaba estrechamente emparentado con la fundación de dicha ciudad. Sin embargo, no se descarta que los españoles hayan encontrado un viejo camino Quimbaya que les haya servido para abrir el propio, pues el mismo Friede menciona en su texto Los Quimbayas bajo la dominación española (1982) que estos mantuvieron intercambios con otros pueblos, y muy probablemente con algunos del otro lado de la cordillera.

Cuando se inició el tránsito por esta ruta, el transporte se efectuaba por medio de mulas e indígenas cargueros o "silleteros", los cuales se emplearían hasta finales del siglo XIX y comienzos del XX. Durante casi cuatro siglos el camino tuvo muy pocas mejoras, y excepto por las "trochas" de pantano por donde escurrían las aguas bajadas del páramo, que cada vez se hacían más pronunciadas y peligrosas, el camino varió muy poco.

Si bien el camino de Herveo tuvo inicialmente un mayor tránsito, gracias al florecimiento simultáneo de las minas de oro de Anserma y Mariquita, el camino del Quindío fue la ruta más importante durante las guerras de pacificación contra los Pijaos (1570-1618) al cerrarse la ruta por el páramo de Guanacas.

Por la necesidad que adquirió en esos años el camino del Quindío para el desarrollo de la actividad comercial, la Corona creó algunos beneficios especiales, sobre todo para la ciudad de Cartago, como los derechos que se cobraban en el paso del río La Vieja y el río Cauca. Las Penas de Cámara se le otorgaron desde 1584 y se renovaron en 1590. En 1588 se autorizó el cobro de dos tomines a los comerciantes que se dirigían a Ibagué por el camino del Quindío.

Cuando los españoles lograron penetrar en el sur de la cordillera pacificando a Timaná y La Plata, reabrieron la ruta por Guanacas. Esto es lo que ha dado pie a afirmar que la ciudad de Cartago se vino abajo: "Desgraciadamente para Cartago y para el camino del Quindío la rebeldía y la extensión de la población indígena, la escasez de esclavos y negros y la rudimentaria tecnología, provocaron la caída vertical de la producción aurífera a finales del siglo XVII. Con ella vino el cierre de la Fundición... a comienzos del siglo XVII, fue la pacificación de Timaná y La Plata, lo que dejó expedita la apertura de un camino directo entre Popayán y Santafé, y provocó el desvío a la mayor parte del comercio de Popayán por el camino de Guanacas" (F. Zuluaga 1995:166). Y a estos hechos también habría que sumarle que "en 1636 se cerró la Fundición y Caja Real, tanto en Cartago como en Cali, y se trasladaron a Popayán" (1995:166).

No desconocemos que esta fue una de los hechos que afectó a Cartago, pero ella misma comprueba que si la ciudad hubiese dependido del camino para subsistir habría sido el momento de desaparecer. Sin embargo, vino la reubicación de la 
ciudad a orillas del río La Vieja entre los años de 1670 y 1691, que ayudó a mantenerla a flote al cambiar las actividades comerciales por otras de índole agropecuaria. Se iniciaba así la etapa de los hacendados y estancieros para Cartago.

Como ya se ha dicho, en el siglo XVIII la ciudad adquirió una especie de resurgimiento con la aparición de las minas del Chocó, pues Cartago basó su desarrollo abasteciéndolas de productos agrícolas y pecuarios, además de servir de intermediaria en el tránsito de mercaderías procedentes de Santa Fé de Bogotá y provincias del oriente que se dirigían hacia dichas minas; la ciudad también se convirtió en un eje importante de abastecimiento de mano de obra esclava. Todos estos hechos lograron que Cartago tomara fuerza en la región constituyéndose como un punto importante en la ruta comercial entre Santafé y Popayán, y de Cartagena hacia Quito y Lima, con lo cual el camino del Quindío protagonizaría el rol principal en las rutas terrestres de estos años.

Así lo anota Francisco Zuluaga: "El surgimiento y crecimiento permanente de las exploraciones en el Chocó (1685-1720, como lo anota Colmenares, Popayán, una sociedad esclavista) no sólo rescataron a Cartago de la ruina, sino que revitalizaron el camino del Quindío, haciendo de él una vía necesaria para algunas remesas de oro hacia Santafé y, fundamentalmente, la ruta de introducción legal de esclavos desde Cartagena hacia el Chocó y toda la Gobernación de Popayán" (1995:166).

Por esto mismo Cartago recibiría algunos beneficios derivados del camino, como lo eran los impuestos de peaje y portazgo, alcabalas sobre transacciones comerciales, y un flujo frecuente de individuos que pasaban por la ciudad. Hay que agregar que durante el siglo XVIII la corona de los Borbones introdujo como una política esencial construir caminos para el mantenimiento del imperio, con ello se beneficiaría el camino del Quindío por las mejoras que se le hizo, pero de paso constriñó a los cartagueños porque éstas debían salir de sus bolsillos y no de las arcas reales.

Claro que no puede olvidar que en todo este contexto comercial, que supone un intenso tránsito de mercaderías por el camino del Quindío y otros caminos reales, existía una precaria economía en la cual la pobreza fue su corolario. La circulación del oro en polvo creó una economía inflacionaria que estrechó los circuitos comerciales hasta prácticamente asfixiarlos (Barona 1995:108-109). El mal estado de los caminos reales como el del Quindío fue una constante durante todo el periodo colonial y aun en el siglo XIX. 


\section{EL ESTADO DEL CAMINO EN EL SIGLO XVIII}

Por la documentación se conocen algunas de las condiciones del camino para el siglo XVIII. El 3 de diciembre de 1754, por ejemplo, el cabildo de Ibagué envió al virrey Solís una representación por medio de la cual se ponía en conocimiento que: “... esta miserable ciudad (Ibagué) sólo se mantiene de los sujetos que transitan una montaña que llaman Quindío la que resulta su traspaso a las provincias del Chocó, camino sumamente bravísimo para aquellas Provincias como para las de Popayán, Buga, Cali, Cartago... el cual no se transita como pudiera a causa de hallarse los caminos rodados y descompuestos, motivo de no haber en esta ciudad sujetos de adelantado caudal que pudiesen capitular con su Majestad el referido camino"(1995:166) $)^{8}$.

Cuatro años más tarde se reafirmaba que a pesar de traer sal, ajo y otros alimentos por dicha ruta, su estado era tan cenagoso e intransitable que sólo la urgencia precisaba viajar por ella (1995:166-167)". Ese mismo año de 1758 "Luis López de la Vega y Juan Gómez de la Cruz hicieron postura de capitulación para que se les asignara el camino, comprometiéndose a mantenerlo limpio, concediéndosele otras cosas: dominio y propiedad del camino, dos leguas a cada lado del camino para obtener materiales para el mantenimiento del mismo, que ninguna persona podría avecindarse ni poblar en las dos leguas de contorno señalado"(1995-168).

Albeiro Valencia Llano en su trabajo La apropiación de la riqueza en el Gran Caldas (1987), también muestra la preocupación del cabildo de Cartago por el camino. En 1776 se ordenó "abrir la montaña del Quindío hasta el alto del Boquerón del Páramo" (Valencia 1987:102) ${ }^{10}$. Con el fin de concertar trabajadores para la obra, a los mulatos de Buga y Cali se les ofreció como aliciente relevarlos en forma perpetua de pagar requintos, incluyendo a sus hijas; así mismo, se les ofreció gracia de tierras a los que quisieran poblar las tierras colindantes, a excepción de aquellas que tuvieran minerales; además se les daría una vaca y un toro a cada familia, también se les otorgaría herramientas y se les daría de comer durante la apertura de dicho camino $(1987: 102)^{11}$.

\footnotetext{
${ }^{8}$ La referencia de archivo fue tomada de: AGN, Colonia, Mejoras Materiales, T. 9, folios 1-2.

${ }^{9}$ La referencia de archivo fue tomada de: AGN, Colonia, Mejoras Materiales, T. 9, folio 20.

${ }^{10}$ Citado por Fabiola Estrada (1998:29).

${ }^{11}$ Ibid.
} 
Para este tiempo el camino ya parecía toda una empresa que proveía ganancias. Es por este motivo que se inició una disputa para arrancarle a Cartago su dominio sobre el camino. "En 1771 Miguel de Viana enfrentó a los jueces de Anserma por la apertura del camino, sustentando que sólo es competencia de la jurisdicción de Cartago. En Ibagué también existía la preocupación por mantener abierto el camino del Quindío, pues de ello dependía parte de la actividad económica de la ciudad. En 1775 el cabildo propuso construir hospedajes para los viajeros en el sitio de Toche, donde además debían construir viviendas para quienes asumieran mantener el camino transitable, propuesta que se aprobó dando inicio a una incipiente colonización de las montañas quindianas desde Ibagué" (Estrada 1998:29) ${ }^{12}$.

Desde este momento se inició una política que perduraría hasta el siglo XIX, la cual sostenía que el poblamiento del camino era beneficioso para el mismo, porque de esta manera se tendría mano de obra inmediata para las reparaciones, y sitios de abastecimiento para los viajeros durante todo el curso de la ruta. Que esta política de doblamiento haya fracasado no indica que el camino no tuviera un flujo considerable de viajeros, pues ningún colono se arriesgaba a vivir en las montañas por la "soledad", además sentía que estaba expuesto a peligros. Si el flujo de viajeros hubiese sido más alto, ofreciendo garantías de subsistencia y lucro, es muy probable que el proyecto de poblamiento hubiese tenido otro desenlace. De hecho, para 1780 los aportes de los comerciantes y de los viajeros fueron insuficientes para garantizar su mantenimiento, y entre los años de 1785 a 1786 la maleza hizo intransitable la ruta invadiendo al camino por completo.

Lo expresado por don Manuel Antonio del Campo y Rivas al virrey Caballero y Góngora en el año de 1785, reafirma que el mantenimiento del camino no fue bueno ni continuado, ya que se menciona una vez más la necesidad de realizar la apertura del mismo y dar vía libre al proyecto de poblamiento como una salida para el mantenimiento de la ruta: "Si a más de la composición del camino que urge, se adaptase el proyecto adaptado para Sierra Morena poblando ancha montaña, sería de grandes ventajas, pues se evitaría a sus traficantes el costo de incomodidad que padecen de cargar todo el viático... si que no omito hacer presente a vuestra excelencia que por la fertilidad de dichas tierras, aguas saludables gratas; climas benignos y por la abundancia que hay de gente pobre y labradora contribuyendo con alguna ayuda de la costa no faltarían familias en Ibagué y Cartago que la poblasen y se estableciesen fabricando casa o pieza de alojamiento, y ayudasen de

${ }^{12}$ Fuente documental Archivo Histórico de Cartago, paquete 1776, legajo 32, folio 5v. 
reparar y componer el pedazo de camino de jornada a jornada que se les encargase"(F. Zuluaga 1995:168) ${ }^{13}$.

Durante estos años de preocupación por el camino del Quindío, se dio un hecho de vital importancia para quienes aún hoy les interesa conocer su ruta exacta; se trata del documento "La Medida". El documento es referenciado por el historiador norteamericano Larry Vito Larrichio (2003) y es importante porque se trata de un reporte a la corona Borbona sobre el progreso de la renovación del camino, hecho por un hombre llamado Buenaventura quien midió la distancia completa entre Ibagué y Cartago, logrando así un cálculo preciso del camino: “i 20 leguas, 1531 varas y 24 pulgadas de "plaza a plaza!" Pero lo más interesante del documento de Buenaventura es que da una lista de 86 secciones del camino, incluyendo el trozo original del mismo entre Filandia y Cartago, sección que se consideraba como la parte del "eslabón perdido", la variante de la Trocha, raíz del pleito entre Cartago e Ibagué en 1777. La relevancia del documento también está relacionada con los datos que ofrece para identificar el trozo original del camino y establecer su integridad histórica ${ }^{14}$. El gran aporte de Larrichio al estudio del camino del Quindío radica en el hecho de que identificó 34 de los 86 sitios nombrados por Buenaventura; a partir de ellos se podría trazar la trayectoria histórica que siguió el camino. Esto es muy importante considerando que La Trocha ha desaparecido con los años y que muchos de los caminos reales también se los ha tragado el monte, a pesar de que fueron durante tanto tiempo los ejes sobre los cuales giró la vida de nuestro país.

Buenaventura describió el viaje que hizo por el camino, y dijo que regresó “con felicidad de la cordillera del páramo del Quindío, de la vista de la apertura de esta montaña. De mi pertenencia: con ingenuidad, y verdad aseguro a Vuestra Excelencia ha sido uno de los mayores gustos de mi vida". También hizo comentarios sobre la excelente condición del camino y tomó registro sobre algunas especies naturales que podrían ser "plantas útiles".

Larrichio señala que cada uno de los 86 sitios registrados por Buenaventura, identificaban mojones ecológicos a lo largo del camino, como ríos, quebradas, cuchillas, boquerones y estructuras hechas a mano, entre ellas puentes y tambos. Los sitios que a continuación se marcarán en negrilla son los que Larrichio

${ }^{13}$ La referencia de archivo fue tomada de: AGN, Colonia, Mejoras Materiales, T. 22, folio 20.

${ }^{14}$ El reporte "La Medida" de Buenaventura al Virrey sobre su éxito en la apertura del camino. "Abrir camino nuevo (la Trocha) y viejo (Serrillos). 19 de Mayo de 1777. AGN, Sección colonia, Fondo Mejoras Materiales, Tomo 3, folios: 275-278. 
identificó aún hoy referidos a nombres de veredas, quebradas, lomas y otros accidentes geográficos:

Buenaventura sale: PLAZA DE IBAGUÉ - Río Coello - Río Combeima Tambo $1^{\circ}$ (la cuesta de San Antonio), Primer alto. / La Palmilla - Yncencial - Tambo $2^{\circ}$ (Cara de Perro) - Quebrada Pontequela - Alto de los Corrales Quebrada de los Corrales - Quebrada Tapias - Tambo $3^{\circ}$ - Moral - Pantano - Quebrada Azufral - Buenavista - Primer Chachafruto - Tambo $4^{\circ}$ (Aguadas) - Segundo Chachafruto - Quebrada Aguascalientes (La ranchería) - Alto de Machín - Tambo $5^{\circ}$ (San Juan) - Puente del río San Juan Pantanitos de Toche - Contadero de alto de Sepultura - Alto Buenavista Yerbabuenal - Bolcita de Yerbabuenal - Tambo $6^{\circ}$ (Tres Cruces) - Quebrada Tres Cruces - Primer Gallego - Quebrada Pie de la Mesa - Pie de la Mesa Alto Mesa del Gobernador - Chorrito de Tochecito - Río Tochecito primera Ceja - Tambo $8^{\circ}$ ("Ceja de en medio) - Ceja Larga - Tambo $9^{\circ}$ (Bolcancito) - Bolsita de la negra - Quebrada Peñón Azul - Quebrada San Rafael - Pie de Paramillo - Paramillo Redondo - Tambo $10^{\circ}$ (Bolcán de los Ajos) - Boquerón del Páramo (El paso del Quindío) - Boca de la angostura larga - Palo Torcido - Cuchilla seca - Magaña - Pringamosal Magañitala Laguneta - Chuscal largo - Chuscal redondo - Palmarcito - Primer Yncecial - segundo Yncencial - Cruz Gorda - Quebrada de Cruz Gorda - Orilla del Río Quindio - $2^{\circ}$ paso del río Quindio - Gramal - $3^{\circ}$ Paso del Río Quindio (aquí el camino desciende la cordillera por donde hoy se encuentra el pueblo de Salento, el cual, no existía en 1777) - Quebrada Boquía (hoy se encuentra la fonda Boquía en los bajos de Salento) - Palmita - Arrayanel - Alto de los Robles (Aquí empieza la ruta de la Trocha) - Cruces - Portachuelo (Actualmente municipio de Filandia) - Socorro - San José - Buenavista Cuchilla los Ángeles (esta cuchilla no queda muy lejos del pueblo de Alcalá, fundado por Sebastián de Marizancena con el nombre de San Sebastián de la Balza en 1792 y el área servía como tambo en el siglo XIX) - Cañabrava Tambo (el único del nuebo camino) - Tomatal - Chorrito - Rancho Ixaca Boca de Guadual - Orilla del Río la Vieja - Piedra de Moler - Quebrada Guadual - Alto Serro Gordo - Alto Bocabajo - Los Posos (desempaladero) Buenaventura llega a la PLAZA DE CARTAGO.

El trabajo de Larrichio basado en el documento de Buenaventura deja ver datos muy valiosos para reconstruir la ruta; además demuestra una primera iniciativa por explorar el territorio, pues Buenaventura hizo hallazgos que serían muy significativos, por ejemplo el de la Palma de Cera que hoy es el árbol nacional de Colombia, descubrimiento que la literatura histórica inglesa le atribuye a Humboldt, pero que Buenaventura ya había registrado, más de veinte años antes que el naturalista alemán. Pese a todos estos significativos esfuerzos por mantener abierto el camino y conocer una ruta exacta de aquél,durante el siglo XVIII el 
camino el camino tampoco presentó un mejor estado, ya que para el año de 1796 se vuelve a hablar de la necesidad de mantenerlo abierto, por lo cual "se firman nuevamente capitulaciones por Pedro Cerezo (comandante de armas de Cartago) y Francisco Villanueva (administrador de correos de Ibagué) para la composición del camino del Quindío" (F. Zuluaga 1995:169-170) ${ }^{15}$.

Este continuado estado de desastre del camino contrasta con una naturaleza maravillosa que cronistas y viajeros describieron con admirados ojos. Dos discursos antagónicos se construyen en el camino: por un lado, el abandono y estado lamentable de la ruta; por el otro, descripciones de flora y fauna jamás imaginados. Pero a veces también el paisaje aparece como un antagonista, el paisaje "incivilizado" que contrasta con el "civilizado". La naturaleza entra en "el reino intensamente humano del valor" y se carga de una subjetividad, de una narrativa propia de los seres humanos (Cronon 2002:37, 41).

\title{
EL PAISAJE RETÓRICO DEL CAMINO
}

Es muy cierto cuando se dice que la naturaleza permanecería muda si no es por los seres humanos. Somos nosotros quienes la hemos nombrado y cargado de intereses y conflictos hasta convertirla en el centro moral de nuestras historias (Cronon 2002:57). Al lado de la retórica determinista del paso del Quindío como una barrera infranqueable, ya expuesta, los viajeros y cronistas de este paso, y los proyectos de mejoras del propio gobierno para integrar la nación, no fueron ajenos a sus propios intereses y conflictos, extrayendo una cantidad de moralejas del paisaje retórico de la ruta y sus provincias para convertirlas en historias.

La exuberancia y enumeración de tantas especies desconocidas fue una de ellas. Esto es lo que diría Pedro Cieza de León (1985:141) de la Provincia de Quimbaya:

\begin{abstract}
Como los cañaverales son tan espesos hay muchas alimañas por entre ellos, y grandes leones, y también hay un animal que es como color parda; la cabeza tiene como zorra; vi una vez una de estas, la cual tenía siete hijos que estaban junto a ella, y como sintió ruido abrió una bolsa que Natura le puso en la misma barriga y tomó con gran pereza los hijos, huyendo con mucha ligereza, de manera que yo me espanté de su presteza, siendo tan pequeña y correr con tanta carga, y que anduviere tanto. Llaman a este animal chucha. Hay unas culebras pequeñas de mucha ponzoña, y cantidad de venados, y algunos conejos y muchos guadaquinajes, que son poco mayores que liebres, y tienen buena carne y sabrosa para comer. Y otras muchas cosas que dejo de contar porque me parecen que son menudas.
\end{abstract}

Cieza dejó de contar tantas cosas porque le parecían "menudas" en una provincia de las más fértiles y abundantes del mundo; tan gruesa la tierra de los

${ }^{15}$ La referencia de archivo fue tomada de: AGN, Colonia, Mejoras Materiales. 
valles adonde no llegaba la arena, que se podía sembrar, con poco trabajo, todo lo que se quisiera. El cronista vio papas, muchos frisoles, raíces de yuca y muchas de otras; unas frutas muy singulares a las que llamaban pepinos, cantidad de árboles de guayabas y de guabas y paltas, que eran a manera de peras; guanábanas, caimitos, piñas; en la espesura de los valles algarrobas algo largas y angostas pero no tan grandes como vainas de habas; también viñas y muchos y grandes higuerales (273-274).

Siglos después Humboldt (1801) también se maravillaría de esta naturaleza y fauna exuberantes. Líquenes y hongos entre sempiternas lluvias donde sólo los cargueros eran útiles, porque en el lodo aguantaban más que las mulas (1982:112). "En Quindío vi verdaderos Lichen icmadophila, Cladunia coccifera, Sphaerina hypoxilon-dice el viajero- así como fósiles, los mismos en todos los climas y tipos de montañas, aún más, tienen afinidades químicas, combinadas y también independientemente. En los valles de los Andes (en las montañas de Quindío, por ejemplo) hemos encontrado una cantidad increíble de musgo de gran follaje, de tal manera que cubre totalmente los troncos de los árboles, los caminos, con una espesa alfombra -y precisamente en los valles calientes, bajo las palmeras Helicóneas..." (113).

Humboldt también vio algunos cultivos de canela Mirystica (Otoba) y pimienta al igual que de quina. En las angosturas del camino, en donde a veces la oscuridad era indescriptible en pleno día, también vio plantas etiorlite. La salubridad por lo tanto no era la mejor en el camino -argumentaba el viajero-, pues además una selva espesa y húmeda cubría una gran cantidad de materiales vegetales, depósitos de piritas que descomponían y estratos de arcilla gredosa (112). En tales condiciones del ambiente y del propio camino (paredes cubiertas de musgo y cunetas llenas de lodo negro) un carguero gastaba mínimo 12-14 días desde Cartago a Ibagué, pero muchas veces 20-25 días (112-113). Aquellas condiciones eran antes de Buenavista; después se vadeaban pantanos y lagunetas, como si se paseara en un corral, en un charco de estiércol. "El lodo negro y líquido en el bosque de guadua casi siempre es de dos pies de profundidad y peligroso por las púas del Arundo Bambus y de la espinosa Vaginae Deciduae, los que lastiman los pies. Los cargueros pasan esos pantanos de guadua muy habilidosamente, sobre delgados juncos de los que cualquiera sin experiencia se resbala" (113).

Pero a pesar de todo Humboldt habla de un tránsito intenso por el camino del Quindío. A cada momento era habitual encontrarse con mulas, bueyes y silleros manteniendo un comercio activo (113). Los accidentes eran comunes, sobre todo porque en el Quindío se utilizaban más bueyes que mulas de carga, y aquellos regresaban vacíos desde Cartago. El mayor uso de los bueyes se debía a que éstos 
en medio de los aguaceros y el lodo aguantaban más que las mulas. Para protegerse de la lluvia, casi dos o tres horas se encontraban lugares desprovistos de árboles, abiertos a hachazos, que se llamaban rancherías o contaderos; pero en realidad toda la ventaja de estos claros radicaba en hallar mejor pasto para los animales (116117).

Para alimentarse, fuera del avío o bastimento (tiras de carne seca de res, bizcochos de maíz, huevos duros, azúcar en bruto -panela-, chocolate, ron, pedazos de sal o "piedras" que resisten a la humedad y cigarros -Boussingault 1994:153-), los viajeros podían recurrir a los frutos de palma, a pesar de que eran escasos; también a los cogollos de palma (las hojas no maduras y más gruesas), nueces y pavas en abundancia (Phasianus y Crax) (Humboldot 1982:115).

La retórica del paisaje descrito por Humboldt demuestra, sin duda, que el trayecto del camino fue realizado en pleno invierno. Imagen que contrastaría con otras en las que se mencionaría que el estado del camino era muy bueno. El viaje fue tan penoso para Humboldt y sus acompañantes, que debieron acampar algunos días en Boquía porque las hojas de bijao ya no eran funcionales (116). Estas hojas eran imprescindibles para todo viajero. Se empleaban tanto en la ruta para protegerse de la lluvia como en los contaderos donde cada viajero tenía que hacer su "casa". Mientras los jóvenes buscaban bejucos en el bosque (plantas trepadoras, Aristolochias Bignonias), los cargueros mayores cortaban palos. Los ranchos eran amarrados con bejucos o en su defecto con pita o fique Agave; por último, se cubrían con las hojas de bijao a manera de tejas (Novus Genus Munandriae). Humboldt describió así tan útiles hojas:

Crece en los ríos afluentes del Magdalena, muchas veces también en la caída occidental de los Andes, en el río Quindio, La Vieja y entre Cartago y Buga. Las hojas de largos tallos, de 18-20 pulgadas de largo y de 12-14 pulgadas de ancho, son preparadas antes de salir de viaje haciendo un incisión o doblez en el nervio de la hoja. Valiéndose de este doblez el carguero cuelga las hojas a manera de tejas, en los hilos o bejucos con los que está amarrado el armazón. Todas estas son viejas artes de la experiencia indigena, aprendidas por los españoles. Es comprensible cómo esos techos de hojas, mejor que cualquier carpa, resisten a los fuertes aguaceros... El envés de las hojas (parte inferior-andicans- tiene una capa blanca y jabonosa, de brillo plateado, que le impide la penetración del agua. En cuanto las hojas están secas, se pela ese barniz (así lo llaman los habitantes) y eso es señal de que hay que apresurarse a dejar la cordillera porque el techo ya no aguanta agua. La casa es tan espaciosa que caben dos catres. Debajo y al lado de aquellos habitualmente duermen con nosotros cinco-seis cargueros (115).

Un poco más de cincuenta años después, Isaac Holton (1857) también se referiría a estas hojas de bijao (Heliconia bijai). Hoja de la forma característica de la canna de los jardines y de la mata de plátano, de dos pies de largo, blancuzcas por debajo, y empleadas para hacer los techos (1981:395). Holton también habla de 
la utilidad de estas hojas como abrigo: "Antes todos los peones y cargueros tenían que llevar su porción de Bihai cuando viajaban al oriente, y el caminante dormía casi quince días bajo ese techo transportable" (135).

En su recorrido por el camino del Quindío, Theodore Gaspard Mollien (1823) también se refirió al número incalculable de especies, contrario de África en donde "a medida que uno se aleja de las soledades habitadas por los muros, al norte de Senegal y del Dialliba, su número tiende a disminuir" (Gaspard 1992:350). De igual forma, el viajero mencionó los volcanes de la cordillera y en especial los lagos de los páramos donde se pescaba el chimbi, una especie de morena (350).

Charles Stuart Cochrane, otro viajero de la ruta, tampoco dejó de mencionar la exuberancia de la naturaleza, en especial de los cultivos con grandes posibilidades de comercialización (cacao, café, caña de azúcar, tabaco y plátano). Al igual que Humboldt, el viajero prestó especial atención a los contaderos (lugar donde cuentan las mulas), y en uno de aquellos, llamado Calejo, en La Línea, levantaron el rancho debajo de una palmera donde había papagayos que eran de buena comida, y prendieron una gran hoguera para evitar los tigres que perseguían especialmente la carne de perro. Según el viajero, los tigres pequeños jamás atacaban al hombre, pero seguían a los arrieros durante días esperando que algunos de ellos muriese (1994:260). Sin embargo, lo que más le llamó la atención en la ruta a Cochrane fue la honradez de la gente, pues el día que tropezó con el hombre del correo (que hacía la ruta de Ibagué a Cartago en cuatro días) iba muy despreocupado, pese a ir cargado de oro y platino (272). Para Cochrane el viaje no fue tormentoso como para Humboldt; la diferencia de las dos retóricas es evidente.

Tanto Humboldt como Cochrane describieron la ruta del camino desde Ibagué hasta Cartago. Sin embargo John Potter Hamilton (1824), otro viajero de la primera mitad del siglo XIX, lo describió en sentido contrario. Para entonces -señala el viajero (1993:322) - cuatro caminos convergían en Cartago: el que partiendo de oriente conducía a las provincias de Mariquita y Bogotá; el que arrancando hacia el occidente llevaba a las ciudades de Ataria y Novita; hacia el sur el que iba hacia Popayán, Pasto y Quito, y por último el que comunicaba a la provincia de Cartago con la de Antioquia.

Según el viajero, en Cartago se podía comer muy buen por la harina que introducían de Bogotá a través del camino del Quindío (330). Debemos entonces imaginar que aprontaron una buena cantidad de pan antes de emprender el viaje, que sólo saliendo de Cartago auguraba lo peor, pues por tres cuartos de legua encontraron en muy malas condiciones el camino y después de seis horas de viaje debieron acampar en una casa solitaria en las márgenes del río La Vieja (337). 
Hamilton estaba decidido por ningún motivo a montar a espaldas de un hombre, a excepción de que enfermara y quedase a merced de los tigres (324).

Como los anteriores viajeros, Hamilton también quedó admirado con la exuberancia de la flora y la fauna del Quindío: saliendo de Cartago patos salvajes, cercetas y becardones en los pantanos; la hormiga cazadora de gran tamaño que atacaba a los sapos y a las culebras pequeñas $(330,332)$. A medida que ascendía quedó impresionado por las selvas impenetrables, y en ellas el temerario rugido del tigre acompañado del desapacible aullido de los simios rojos y el áspero graznar de las aves nocturnas hasta convertir aquellos sonidos en una serenata infernal (341). "iQué campo de investigación tan amplio y rico ofrecían estas montañas exclamaba el viajero al ver la enorme cantidad de pájaros tan raros-, pero eso sí arrostrando toda privaciones y penalidades" (342). Culebras, entre ellas una de piel verde brillante y de ocho pies de largo que un peón mató cuando yacía dormida; animalillos de toda clase y un jaguar que estaba bebiendo a la orilla de un arroyo; pavos silvestres y muchas huellas de tigre y de oso negro; también huellas de dantas (asnos salvajes) de pezuña hendida en dos y, según la descripción de los peones, de color leonado oscuro, muy veloces y que podían alcanzar mayor tamaño que el de un asno bien desarrollado (346). En fin, mariposas de todos los tamaños, algunas muy grandes; micos descolgándose de los árboles y haciéndoles muecas desde los árboles. Pero todo -enfatizaba Hamilton- a punto de perderse para los científicos si el gobierno no ponía pronto remedio al camino, porque la naturaleza comenzaba a ensoñorearse nuevamente (348-350).

Entre todos estos viajeros, tal vez fue Holton el que utilizó más adjetivos en su retórica del paisaje. Un camino que seguía la ruta de doscientos años atrás, y el que en ese momento (1857) estaba siendo reparado por presidiarios (1981:110). ¡Ningún cambio importante se había hecho en dos siglos! -exclamaba el viajero a su paso- "apenas pude creer mis ojos al leer en dos piedras planas inscripciones que muestran que este camino tiene más de doscientos años... La primera dice: 'Por aquí paszó (sic) Francisco Peñaranda, a 24 de agosto, 1641”(380, 383).

"En la orilla de una arroyo encontré un magnifico ejemplar de cola de caballo de cinco o
seis pies de altura -dice el viajero- para referirse a una especie desconocida a sus ojos
(391). "Nunca en un camino transitado habia visto tal soledad, si es que puede hablarse de
soledad cuando se escucha el canto de las aves, entre otras de pavos y un bello tucán verde
brillante" (380). Al parecer el bastimento del viajero y sus acompañantes no era bueno,
porque "afortunadamente el hombre que vivía en esas soledades había matado un oso negro
y nos vendió su carne" (387).

Holton encontró lodazales, callejones y trampas temerarias a lo largo del camino, pero cuando llegó a Boquía dijo que llegó a un camino hermoso (392), y cuando por primera vez divisó la panorámica del valle del Cauca no pudo más que 
hablar de su color vivo verde maravilloso en contraste con las llanuras secas de Ibagué y El Espinal (395).

Lo cierto era que en todas aquellas maravillas y soledades, coincidían los viajeros, había todo un campo de potencialidades y de tierra por poblar.

\section{COLONOS, PRESIDIARIOS Y VAGOS EN LOS PROYECTOS DE POBLAMIENTO DEL CAMINO EN EL SIGLO XIX}

El ejemplo más claro del proyecto de poblamiento del camino del Quindío, y en cierta medida de mayor éxito, fue el llevado a cabo por Sebastián de Marisancena, el cual tomaría el nombre de San Sebastián de La Balsa y posteriormente Alcalá. Esta fundación tenía la intención de fortalecer los intereses privados de Marisancena en el Comercio de la Carrera de Indias y apropiarse de las tierras baldías por donde pasaba el camino; tal vez por tratase de un interés en gran parte económico fue que subsistió esta población a la que en más de una ocasión su fundador tuvo que inyectarle recursos e ingentes esfuerzos para que no desapareciera. Marisancena fue un influyente vecino de Cartago que mantenía negocios en todas las provincias que se extendían hasta Ibagué. Esto es lo que dice Fabiola Estrada (1998:36) de dicho proyecto:

Sebastián de Marisancena en 1791 recibe de la corona el título de Juez Poblador, esto implicaba que podia organizar y dirigir el poblamiento hacia las montañas del Quindio. Para hacer efectivo el desplazamiento de personas, se creó una lista donde se matriculaban los interesados, y posteriormente se aceptaba el traslado conjuntamente con sus respectiva familia. Fueron cerca de 39 inscritos, de los cuales 29 eran casados (...) Marisancena debia garantizar a los matriculados los alimentos, herramientas y animales para que cada familia comenzara a instalarse, además de asignarle un pedazo de tierra para que trabajaran y construyeran su vivienda (Estrada 1998:36).

Muchos de los pobladores iniciales de La Balsa huyeron y Marisancena se encargó de perseguirlos, así como de apoyar a los que se quedaron. Un sinnúmero de inconvenientes surgieron en el establecimiento de la población, pero fue su fundador quien se mantuvo firme en no dejar que desapareciera. Una de esas medidas la tomó hacia 1805 cuando solicitó a las autoridades eclesiásticas, con sede en Popayán, el nombramiento de un sacerdote que administrara religiosamente a los vecinos de La Balsa. Marisancena nunca logró que se designara al cura y durante los años de 1826 y 1827 se desató de parte de las autoridades eclesiásticas una batalla legal contra él "por no existir ya la población de La Balsa, por haber sido demolida su iglesia y por llevarse sus pertenencias, se le acusó de haber profanado lo que era de la Iglesia" (1998:50). Pese a todo, San Sebastián de la Balsa continuó existiendo en condiciones cada vez de mayor aislamiento y sin iglesia durante las décadas restantes del siglo XIX (1998). 
Otro proyecto para el poblamiento del camino durante el siglo XIX fue el plan de don Ignacio Durán, quien proponía una colonización pero por la otra ruta, es decir, a partir de los Cerrillos o Cerritos como ya se le empezaba a llamar a este sitio:

Principiará la medida del camino desde el confin del pueblo de Los Cerritos, allí se podrá un término de piedra fijo la milla, otro con su marca primera milla, $2^{a}, 3^{a}$ hasta la legua, y con el mismo orden hasta las tres leguas donde se pondrá otro término. En el centro de las tres leguas se fabricará la iglesia, casa para el cura, vecino, hospedería y tambo. Al cura se le dará una cuadra de solar con iglesia y casa, al vecino otra con casa, albergo para los pasajeros y tambo para apostar cargas y bagaje. A más de la cuadra de solar se les consideraba una legua de tierra cuadrada de labor, pero fuera del camino, tanto al cura que al vecino. A la mitad de la primera y tercera millas de la primera legua se colocarán los vecinos, a quienes se les hará el mismo reparto, y con este método se harán las distribuciones de parroquias y vecinos hasta los términos de Ibagué... Todos los vecinos en su respectivo hogar harán un potrero para encerrar las caballerías... mantendrán también en buen estado la hospedería y tambo en beneficio de los pasajeros (F. Zuluaga 1995:170).

Así, en 1804 el mencionado Ignacio Durán, en un comienzo en compañía de Pedro Cerezo y Juan José Salamandro y posteriormente solo, presentó un plan para la Apertura y Colonización del Camino del Quindío. Este plan -quizás el más completo, detallado y racional encontrado en los documentos, dice Francisco Zuluaga-, "aunque fue muy bien recibido por el virrey y el fiscal, sólo se aprobó en 1807. Esta dilación se debió a las prolongadas consultas a los cabildos y la interpolación de don Sebastián de Marisancena, quien veía lesionados sus intereses y los de su colonos de San Sebastián de La Balsa, o al menos así lo daba a entender" (1995:170), pues como ya hemos dicho Marisancena había solicitado permiso para hacer una parroquia de vecinos en el sitio de La Balsa desde 1791. "El plan de Ignacio Durán proponía que, llegando a la confluencia del río La Vieja y la quebrada Piedra de Moler, se construyera un camino por terreno firme que, pasando por el pueblo Pindaná de los Cerrillos, cayera a Cartago"(1995:170). Esta ruta la consideraba Durán más segura para el comercio y para la acción colonizadora, pues desde que corrieron rumores de apertura del camino "varias familias indígenas dispersas en la selva habían retornado a su antiguo resguardo"(1995:179).

En 1808 don Pedro Cerezo, administrador de correos de Cartago, en compañía de otros dos funcionarios públicos de la ciudad, volvió a solicitar la apertura de la montaña del Quindío, sugiriendo en esta oportunidad que se abriera por la vía de La Balsa en vez del camino de Cartago Viejo. "Es de esperarse que, por las guerras de independencia, ni don Ignacio Durán pudo adelantar su plan de apertura por Pindaná de los Cerritos, ni don Pedro Cerezo logró la restauración definitiva de su camino por San Sebastián de La Balsa. La guerra de independencia se encontrará 
con un camino tan fragoso, enmontado e intransitable, como lo fue durante la colonia"(1995:170).

Así pues, cuando se estaba a punto de librar las guerras de independencia el camino del Quindío presentaba los mismos problemas de siempre. De igual manera el proyecto que aportaría la joven República para solucionar los problemas del camino del Quindío parecieron ser los mismos: mejoras y poblamiento.

No obstante, para mediados del siglo XIX el camino se constituyó finalmente como institución quedando matriculado a la Secretaria de Relaciones Exteriores y Mejoras Internas. El gobierno dispuso entonces que la cárcel del Tercer Distrito, ubicada en Cartago, fuera la encargada de llevar a cabo los proyectos de mantenimiento y mejoramiento del camino del Quindío. En una relación de los gastos extraordinarios decretados por la gobernación del Cauca para la conducción y mantenimiento de los vagos de enero de $1844^{16}$, se muestra claramente el vínculo que existía entre el camino y el presidio. De igual manera encontramos que el gobierno reiteró el proyecto de poblamiento que a finales del siglo XVIII y principios del XIX se había dejado en manos privadas, pero esta vez lo veremos aplicado en los reos que iban a trabajar al Quindío procedentes de sitios tan diversos como Panamá, Pasto y Socorro, a quienes se les motivó para que se "quedaran en las montañas del Quindío". El Cuadro 3 que muestra la ocupación del presidio $^{17}$ :

Cuadro 3

\begin{tabular}{lccc}
\hline \multicolumn{1}{c}{ Altas } & Presidiarios & Bajas & Presidiarios \\
\hline $\begin{array}{l}\text { Remitidos por la provincia del } \\
\text { Cauca en razón de sus condenas. }\end{array}$ & 1 & Por cumplimiento salieron & 3 \\
$\begin{array}{l}\text { Remitidos por la provincia de } \\
\text { Pasto en razón de sus condenas. }\end{array}$ & 1 & Muertos & 0 \\
Reincorporados después de fugas. & 0 & Prófugos. & 0 \\
Por otra clase de entradas. & 0 & Por otra clase de salidas & 0 \\
\hline
\end{tabular}

La conclusión del cuadro decía así: "El día $1^{\circ}$ del mes existen 66 hombres en E. Que comparados con 63 que existen hoy resulta la diferencia de altos y bajos que aparece en el cuadro. Los 65 presidiarios se hallan distribuidos en estos términos:

\footnotetext{
${ }^{16}$ CDIHR, Fondo Gobernaciones Varias. Legajo 094, Folio 613.

${ }^{17}$ CDIHR, Fondo Gobernaciones Varias. Legajo 094, Folio 621.
} 
trabajando en el camino del Quindío, 27; trabajando en el Hospital, 36; trabajando en concierto en esta provincia, 2 "18.

La legislación expedida por el Gobernador de la Provincia del Cauca, Jorge Juan Hoyos, amparándose en el Artículo $5^{\circ}$ del decreto del 6 P. E. de 31 de Marzo de 1843, solicitaba concertar vagos para trabajar en la obra del Quindío, cuya dirección, conducción, mantenimiento y custodia estaría a cargo del presidio del tercer Distrito; serían contratados capataces que tendrían un sueldo de diez pesos mensuales cuando hubiese más de doce vagos en la obra; el director debería expedir los gastos para el mantenimiento de los vagos del presidio y de los presos, sin confundir ambos balances; los vagos estarían sujetos a ciertas condiciones como:

- Trabajar por el tiempo de la condena sujetos a las órdenes del director del presidio.

- Estar sujetos al mismo sistema de corrección de presidiarios, por lo que se les podía aplicar los máximos castigos.

- Si intentaban fugarse perderían el tiempo que llevaban pagado de su condena.

- Recibirían ración todos los días, incluso los feriados, y serían asistidos en sus enfermedades y vestido lo mismo que los presidiarios.

- Si se manejaban bien los dos primeros meses de la condena serían premiados con una mejor paga dándoles lo que se le acostumbraba pagar a un peón libre concertado.

- Si llevaban seis meses sirviendo en la obra a satisfacción, "i quienes después de este tiempo fijarónse en la montaña como pobladores de ellas, se les dará una montaña entera para que cultiven y hagan su casa además una hacha, una harada i un machete, con la obligación de mantener la labranza suficiente para la manutención de cuatro peones por lo menos, sembrando lo que les provenga por el director del camino"19.

- Si cuidaban bien de la labranza y se les cumplía el tiempo de la condena, se les darían todas las ventajas que tenían los pobladores por decreto del 13 de julio de 1842. Lo mismo se haría al considerar la apertura del camino,

\footnotetext{
${ }^{18}$ CDIHR, Fondo Gobernaciones Varias. Legajo 094, Folio 621.

${ }^{19}$ CDIHR, Fondo Gobernaciones Varias. Legajo 094, Folio 555-556.
} 
siempre y cuando antes de dos años presentaran labranza en buen estado como se les concedió.

- Mientras no se formara una población en el Quindío, los vagos, cuyo destino era formarla, seguirían trabajando en la obra a ración y un sueldo ${ }^{20}$.

Es evidente que el proyecto de mejoramiento del camino impulsado por el gobierno, consistía en emparentar el presidio con el camino para el poblamiento de la zona. No obstante, también se involucró a todos los habitantes por medio del servicio personal subsidiario. Este punto se observa en la notificación enviada al secretario de E. En el D., del interior, en donde la población de Anserma Nuevo expresaba su insatisfacción, porque "desde el año pasado (1843) e gracias al decreto del 27 de marzo del mismo año, sobre composición i mejora del camino del Quindío gravita sobre nosotros el insoportable poder legal de tener que prestar el servicio subsidiario en la montaña del Quindío"21.

De otro lado, había ciertos gastos que dependían del gobierno central porque todas las comunicaciones de presupuestos y peticiones eran enviadas a él para que las resolviera. Son constantes los "informes de gastos" y "peticiones de materiales", entre los cuales se evidencia que los gastos como tal, aunque no podían ser cubiertos por el gobierno, no pasaban de ser los sueldos de agregados, concertaciones, directores y capataces que trabajaban en la obra; los materiales que se pedían no eran muchos tampoco, en su mayoría herramientas rudimentarias de escasa tecnología, como picas, azadones y machetes.

El gobierno vio la importancia de promocionar las obras, pues se buscaba mejorar la comunicación en un momento crucial para la unidad política ante la reciente independencia. El camino pasa a jugar el papel "de factor de cohesión política", como queda indicado a continuación: "Grandes ventajas traerá sin duda a la República el mejoramiento del camino Quindío. Las provincias del sur cosecharán óptimos frutos de tan útil empresa, i un punto de comunicación con la capital de la República, no será el menor de su beneficio. El gobierno en general, podrá ejercer una acción más pronta y cumplidamente. Los desórdenes ocasionados por descontentos serán sofocados con presteza. El camino adquirirá un grande incremento disminuyéndose considerablemente el gasto de conducción de mercancías nacionales y extranjeras. La riqueza individual se mejorará y por

${ }^{20}$ Ibid.

${ }^{21}$ CDIHR, Fondo Gobernaciones Varias. Legajo 094, Folio 596. 
consiguiente las ventas públicas se aumentarán. La patria será más grande y mejor. Estas ventajas serán seguidas de otras de no poca consideración",22.

El proyecto de poblamiento por cuenta del gobierno se diferenciaba del privado en que el primero no buscaba colonos, sino que intentaba introducir vagos como pobladores de zonas seleccionadas al borde del camino. Este fue el caso de "el vago Jerónimo Quijano condenado por jefe político del cantón de Panamá, [quien] fue ordenado a formar nuevas poblaciones en el camino del Quindío"23. Como era de esperarse este sistema de poblamiento fomentado por el gobierno tenía sus críticas. Así lo expresaba una comunicación enviada al ministro de E. En el D. del Interior, donde se decía que aunque era importante el poblamiento de las montañas del Quindío, se recomendaba que no se enviaran vagos a tal tarea porque al contrario de poblar, estos se pondrían a atracar a los viajeros, ocasionado más desolación en el camino; por eso era mejor pedir pobladores de Antioquia y el Socorro:

No dudo que la primera contribuya a ésta empresa porque sus hijos laboriosos, acostumbrados a variar de citio i movidos por un hábito adquirido de hacer nuevos establecimientos se han avanzado ya sobre este cantón; pero el número que hasta ahora se presenta no es suficiente para poblar el camino, a lo cual deben ser ellos destinados, por ser gentes honradas que viven en familias, son sembradores $i$ amantes de las tierras frías $i$ de las montañas... El socorro dará también gente para fomentar la población, pero será necesario además de las ventajas ofrecidas utilizar la seducción o la fuerza. Pues lo que hoy nos preocupa es proporcionar recursos a los pasajeros en la montaña $i$ hacer que el camino sea transitado inmediatamente... (...) Consiguiente a éstas observaciones es la necesidad de prevenir que no se manden por revoluciones de provincia, en calidad de pobladores a las mujeres ni a hombres inútiles. Trabajadores es lo único que se quiere, y la obra del camino no puede ofrecer contra la vagancia sino un establecimiento en el que se pueda enseñar a trabajar... I últimamente para alagar a los moradores de la Antioquia, a quienes dose como veinte fanegadas por poco para sembrar en dos años, que se les permita derrocar el bosque que necesiten i convertirlo en pastorales sin pervisión alguna de arrendamiento con tal de que se radicasen ${ }^{24}$.

Testimonios como el anterior también permite comprender más factores que motivaron a la colonización antioqueña; aquí, por ejemplo, se ve que se les concedía privilegio y se fomentaba su traslado.

El proyecto del gobierno de poblar el camino también había sido promovido por el libertador, y se mantuvo durante todo el XIX como una política de estado:

\footnotetext{
22 CDIHR, Fondo Gobernaciones Varias. Legajo 094, Folio 597.

${ }^{23}$ CDIHR, Fondo Gobernaciones Varias. Legajo 094, Folio 613.

${ }^{24}$ CDIHR, Fondo Gobernaciones Varias. Legajo 094, Folio 639.
} 
El gobierno de Colombia toma bajo especial e inmediata protección la apertura de caminos, ofreciendo por su parte cuantos auxilios se necesitasen para la apertura... Se conceden exención de derechos a todos los habitantes que vayan a poblar las cercanías inmediatas del camino, con tal que en las poblaciones que se establezcan funden alli casas y constituyan a ser vecinos de ellos. Esta gracia será por veinte años a contar por el día de la fecha... El intendente del departamento se encargará de dar toda protección al encargado de dirigir los trabajos del camino y de presentarle odas las observaciones que sus conocimientos le ofrezcan para el mejor desempeño de esta empresa... El gobierno de Colombia convida a los buenos ciudadanos de provincia a que cada uno concurra con los medios, a fin de perfeccionar la apertura del camino ${ }^{25}$.

Sobre el poblamiento, los viajeros cronistas que recorrían el camino también se manifestaron. Cochrane (1994:268) respecto a las tierras de aledañas a Cartago dijo:

Por el juez local supe que hay mucha tierra sin dueños, que se podría repartir a colonos, que fácilmente podrían obtener lo necesario para vivir, claro está, sin lograr acumular riquezas, ya que la exportación de lo producido es extremadamente dificil... En los alrededores de la ciudad de Cali, lo mismo que en Ibagué, se podrían cultivar los cereales. La exportación más favorable de esta región hace vía Buenaventura sobre el océano Pacífico; pero aún así, el transporte en mulas cargueras hasta el mar cuesta ocho pesetas por cuatro arrobas. Desgraciadamente, el río Cauca solo es navegable en trayectos cortos, y, como se precipita de vez en cuando a través de estrechos rocosos, no se puede pensar en un transporte por agua, los costos serían imposibles" (1994:266).

Debemos finalizar diciendo que pese a la dura topografía del camino y a la aplicación en éste de incontables proyectos, la mayoría de ellos frustrados, el camino del Quindío fue un importante antecedente para la configuración política del país. El camino fue importante porque articuló todo el eje comercial y político de una región, sirvió de guía de migraciones y colonizaciones y abrió la frontera agrícola con la repartición de baldíos en sus laderas. Durante las primeras décadas del siglo veinte adquiriría relevancia con la exportación cafetera y el despegue de la industrialización, que siguió buscando el Atlántico por la también sempiterna ruta colombiana: el río Magdalena.

Tal vez lo más importante que se deriva de este texto es que al lado de ese paisaje retórico construido por la pluma de cronistas y viajeros, se escribió otra historia aún por desvelar, la de colonos, presidiarios y vagos que poblaron una tierra "libre". Esa tierra que marcó la narrativa épica de la colonización, pero también la de otra narrativa que estaría por elaborarse: la repartición de tierra en un escenario de tensiones y conflictos sociales.

${ }^{25}$ CDIHR. Gaceta Colombia, \# 47, “caminos”. Domingo 8 de septiembre de 1822. 


\section{BIBLIOGRAFÍA}

BARONA, Guido (1995): La maldición de Midas: en una región del mundo colonial, Popayán 1730-1830, Cali, Universidad del Valle.

Boussingault, Jean Baptiste (1994): Memorias, Bogotá, Presencia.

CiEZA De LeÓn, Pedro (1985): La crónica del Perú, 3 ed., España, Rascar.

Cochrane, Charles Stuart (1994). Viajes por Colombia 1823 y 1824: Diario de mi residencia en Colombia, Bogotá, Banco de la República. (Colección de viajeros por Colombia).

COLMENARES, Germán (1987): «La economía colonial Neogranadina 1500-174», en OCAMPO, José Antonio, ed., Historia Económica de Colombia, Bogotá, Fedesarrollo-Siglo veintiuno.

CRONON, William (2002): «Un lugar para relatos: naturaleza, historia y narrativa», en PAlacio, Germán y UlloA, Astrid, editores, Repensando la naturaleza: Encuentros y desencuentros disciplinarios en torno a lo ambiental, Colombia, Universidad Nacional-Instituto Colombiano de Antropología e HistoriaColciencias.

ESTRADA, Fabiola: En camino a la Villa del Samán: Monografia histórica del municipio de Alcalá (1998). Cali, Universidad del Valle.

FRIEDE, Juan (2002): «Historia de la Antigua Ciudad de Cartago», en Historia de Pereira, Bucaramanga, Club Rotario de Pereira.

FRIEDE, Juan (1982). Los Quimbayas bajo la dominación española, Bogotá, Carlos Valencia Editores.

GASPARD, Theodore Mollien (1992). Viaje por la República de Colombia en 1823, Bogotá, Tercer Mundo.

HAMILTON, John Potter (1993): Viaje por el interior de las provincias de Colombia, Bogotá, Presencia (Colección de viajeros por Colombia).

HARRIS, Marvin (1996): Antropología cultural, Madrid, Alianza.

Holton, Isaac (1981): La Nueva Granada: veinte meses en los Andes, Bogotá, Banco de la República.

Hoyos KöRBel, Pedro Felipe (2001): Café: caminos de herradura y el poblamiento de Caldas, Colombia, Tercer Mundo.

HumbolDT, Alexander (1982): Extractos de sus diarios, Bogotá, Publicismo y Ediciones. (Extractos realizados por la Academia Colombiana de Ciencias Exactas, Físicas y Naturales y la Academia de Ciencias de la República Democrática Alemana).

INAC (2003): Atlas de Colombia, 5 ed., Imprenta Nacional de Colombia.

Salazar Montoya, Jaime (2000). De la Mula al Camión: Apuntes para una historia del transporte en Colombia, Bogotá, Mundo Editores. 
LARRICHIO, Larry: «La arquitectura del paisaje topográfico-ecológico y adaptación cultural en el Eje Cafetero» ( XII : Congreso de Historia de Colombia: 2003). Popayán.

ROBLEDO, Jorge: «Descripción de los pueblos de la provincia de Anserma 1539». (Trascripción realizada por el historiador Víctor Zuluaga -2002- del Archivo General de Indias -AGI-, Patronato 28, r-66, folios 30-38, Sevilla, España).

VALENCIA Llano, Alonso (1991): Resistencia indígena a la colonización española, Cali, Universidad del Valle.

Zuluaga GómeZ, Víctor (1998): Crónicas de la Antigua Ciudad de Pereira, Pereira, Gráficas Buda.

ZuluagA, Francisco (1995): «Por la montaña del Quindío: el camino real de Santafé hasta Quito, por la montaña del Quindío», en USECHE LOSADA, Mariano (ed.), Caminos reales de Colombia, Bogotá, Gráficas Ltda. (Caminos Reales de Colombia-Fondo FEN Colombia).

\section{ARCHIVOS CONSULTADOS}

ACMP. Archivo del Concejo Municipal de Pereira.

CDIHR. Centro de Documentación e Investigación Histórica Regional.

AHC. Archivo Histórico de Cartago 